\title{
Comparative Efficacy of Biotic and Abiotic Agro- Commercial Products Against Rotylenchulus reniformis Under Field Conditions
}

\author{
A. A. Farahat , A. A. Al-Sayed " and M. Adam and Shaimaa F. Diab" \\ * Department of Zoology and Nematology, Faculty of Agriculture, Cairo University, Egypt.
}

\begin{abstract}
In a field experiment, twelve commercial agro-products were applied as soil drench and 14 as foliar spray as recommended method of application and dose. The results indicated that Biofertile product was the best which had the highest suppressive effects followed by Algeferet and Perfect applied as a soil drench compared to nematode check. The most effective one of products applied as a foliar spray was Indole buteric acid then Feedchem and Amino strong with no significant differences. The experiments indicated that the SAR commercial materials (Nemakill, Indole-3 butyric acid, Gibirilic acid, Indole-acetic acid, Salicylic acid, Citric acid and Glutamic acid) applied as foliar spray reduced nematode counts and reproduction under all conditions. The Mega NPK recorded the best improvement in plant fresh and dry weights and yield criteria. Our results concerning plant growth and yield were highly significant in foliar application treatments more than soil application and the compounds used to induce systemic acquired resistance exhibited more enhancements in plant growth criteria and yield.
\end{abstract}

Key words: $R$. reniformis, Cowpea, commercial products.

\section{Introduction}

The reniform nematode Rotylenchulus reniformis is an obligate sedentary semi-endo devastating nematode parasite attackes over 300 plant species belonging to 46 families grown in the tropical, sub-tropical and warm- temperate regions of the world (Robinson et al., 1997).

Rotylenchulus reniformis, based on its scientific and economic importance was rated as one of the top ten nematodes in plant pathology (Jones et al. ,2013). In Egypt, it is found through the areas with heavier soil such as sandy loam, sandy clay and clay loam soils. Reasons of Rotylenchulus reniformis huge losses include the lack of available commercial host plant resistance, the ability to survive under adverse environmental conditions in the absence of the host (Robinson et al., 2005). Moreover, Rotylenchulus reniformis has short life cycle, wide host range (no host specificity) and unique parasitic habit. 
In recent years, there has been tremendous increase in public awareness on environment pollution and climate change associated with pesticides toxicity and residues. This resulted in the shift in pest management strategies from chemical era in the late 1980s. Since then, the search for novel environmentally friendly alternatives with which to manage plant-parasitic nematode populations has therefore increasingly important. Several national and international companies have developed and produced numerous agro-biological, chemical eco-friendly compounds, claiming that these commercial materials have nematicidal and plant growth promoting properties. Such materials are natural products, biocides (Montasser et al., 2012; Patil and Sharma, 2016), bio-fertilizers (Youssef and Eissa, 2014; Dar and Reshi, 2017), botanical extracts (Abid et al., 2005; ElNagdi and Youssef, 2013), organic (Aktar and Malik, 2000; Zeeshan et al., 2016) and inorganic fertilizers (Bationo et al., 2006; Mahfoud, 2015), plant growth promoters and systemic acquired resistance inducers (Youssef and Lashein, 2014; Luangkhot, 2016).

Extensive research is needed before they could be used reliably for management of plant-parasitic nematodes. Hence this is an attempt to evolve suitable bio, abio-management approaches through the present study for the control of Rotylenchulus reniformis.

\section{Materials and Methods}

This trial was carried out to design some regimes able to control the reniform nematode and enhance acquired resistance in the infected cowpea plants under field conditions. Experiment was conducted in summer 2016 at experimental area of Nematology Division, Zoology and Agric. Nematology Dept. The experimental plan was a randomized complete block design. The experimental field area was $(8 \times 3.7$ $\mathrm{m}$ ) solarly sterilized, plowed, harrowed, rowed and the seeds of cowpea cv. Kareem7 were planted on the prepared field by hand. The seeds were not treated with pesticides. Weeds were controlled manually at 4 and 8 weeks after planting.

Each treatment was replicated 3 times, each replicate had 3 plants and each plot size was three rows ( $2 \mathrm{~m}$ length). Distance between plants was $60 \mathrm{~cm} \times 30 \mathrm{~cm}$. After the seeds germinated, plants were thinned to one per site. One week after germination each plant at the experimental site was infected with 2000 un-swollen females of $R$. reniformis. After one week from infection, infected plants were treated with the commercial agro-products (biotic and abiotic) according to their recommended method of application and dose. (table 1, 2). Three months after planting, plants were taken off and data of nematode counts in $250 \mathrm{gm}$ soil and on2gm roots were enumerated and plant growth criteria (plant fresh and shoot dry weight) and yield (number of pods, fresh and dry weight and number of seeds) were recorded. 
Table (1): Soil drench treatments and doses of the field experiment.

\begin{tabular}{lc}
\hline Treatment & $\begin{array}{c}\text { Dose } / \text { plant } \\
\text { as soil drench }\end{array}$ \\
\hline $\begin{array}{l}\text { Biofertile } \\
\text { (Mixed bacterial solution) }\end{array}$ & $5 \mathrm{ml} /$ plant \\
$\begin{array}{l}\text { Pseudomonas fluorescens } \\
\text { Serratiamarcescens }\end{array}$ & $5 \mathrm{ml} / \mathrm{plant}$ \\
$\begin{array}{l}\text { Mycorrhiza } \\
\text { (vesicular arbuscular mycorrhiza) }\end{array}$ & $5 \mathrm{ml} / \mathrm{plant}$ \\
$\begin{array}{l}\text { Algeferet } \\
\text { (Marine algae extract +Amino acids+ plant growth regulator + Macro and }\end{array}$ & $3 \mathrm{gm} /$ plant \\
micro elements) & $2 \mathrm{ml} /$ plant \\
$\begin{array}{l}\text { Nemastop } \\
\text { (Plant extracts from Alliums spp. + Titrasulphide Tri- Di- Mono) }\end{array}$ & \\
$\begin{array}{l}\text { Nile compost } \\
\text { (plant residues) }\end{array}$ & $2 \mathrm{ml} /$ plant \\
$\begin{array}{l}\text { Nema clean } \\
\text { (Amino acids) }\end{array}$ & $5 \mathrm{gm} /$ plant \\
$\begin{array}{l}\text { Decka } \\
\text { (Humic acid+ Fulvic acid+ Amino acids+ macro and micro elements })\end{array}$ & $0.5 \mathrm{ml} /$ plant \\
$\begin{array}{l}\text { Mega N/P/K } \\
\text { (N19/P19/K19 + Mg 1\%) }\end{array}$ & $1 \mathrm{ml} /$ plant \\
Perfect (Oxamyl 24\%+ Metalaxyl-M4\%+ Tetramethrin 2\%) & $5 \mathrm{gm} /$ liter \\
\hline
\end{tabular}

Table (2): Foliar spray treatments and doses of the field experiment.

\begin{tabular}{|c|c|}
\hline Treatment & $\begin{array}{l}\text { Dose/ plant as } \\
\text { foliar spray }\end{array}$ \\
\hline $\begin{array}{l}\text { Cal mix } \\
\text { (Calcium and Boron carried on organic and amino acids) }\end{array}$ & $0.4 \mathrm{ml} / \mathrm{plant}$ \\
\hline $\begin{array}{l}\text { Humo plus } 40 \\
\text { (Humic acid + macro and micro elements) }\end{array}$ & $0.4 \mathrm{ml} /$ plant \\
\hline $\begin{array}{l}\text { Amino strong } \\
\text { (Amino and organic acids }+\mathrm{Fe}+\mathrm{Mn}+\mathrm{Zn})\end{array}$ & $0.4 \mathrm{ml} /$ plant \\
\hline $\begin{array}{l}\text { Feed chem. } \\
\text { (Macro and micro elements) }\end{array}$ & $8 \mathrm{gm} /$ liter \\
\hline $\begin{array}{l}\text { UniBor } \\
\text { (Boron carried with vitamins + Gibirilic acid) }\end{array}$ & $0.5 \mathrm{ml} /$ liter \\
\hline $\begin{array}{l}\text { Biogibb } \\
\text { (Gibirilic acid + Ca+ B+ cytokinin) }\end{array}$ & $0.25 \mathrm{gm} / \mathrm{liter}$ \\
\hline $\begin{array}{l}\text { Kenzo } \\
\text { (cytokinine+ 6-Benzylaminopurine) }\end{array}$ & $0.2 \mathrm{ml} /$ liter \\
\hline Glutamic acid & $1 \mathrm{gm} /$ liter \\
\hline Citric acid & $2 \mathrm{gm} /$ liter \\
\hline $\begin{array}{l}\text { Nemakill } \\
\text { (Indolylbutric acid } 0.002 \%)\end{array}$ & $0.2 \mathrm{ml} /$ liter \\
\hline Indole-3 butyric acid & $0.1 \mathrm{gm} /$ /iter \\
\hline Indole-acetic acid & $0.2 \mathrm{gm} / \mathrm{liter}$ \\
\hline Gibirilic acid & $0.2 \mathrm{gm} /$ /iter \\
\hline Salicylic acid & $1 \mathrm{gm} /$ liter \\
\hline
\end{tabular}




\section{Results}

\section{Effect on nematode population}

\section{a. Soil drench treatments}

Data presented in table (3) reveal that remarkable significant reductions were apparent in root, soil and final population among most treatments compared to check. The microbial treatments demolished significantly root, soil, final populations and the subsequent build up.

Biofertile was the uppermost with significant differences in reducing nematode soil and root populations and build up among bio-agents and other treatments as well. Transparent significant reductions in nematode criteria were visible in Serratia marcescens, Pseudomonas fluorescens and Mycorrhiza, respectively.

Concerning plant extracts, Algeferet was in the lead and raised steadily its efficacy in reducing the nematode population up to $64.59 \%$ in the field. Meanwhile, Nemastop and Nile compost behaved the same with no significant differences.

In view of amino and organic acids, Nemaclean and Decka, they were not significantly different in action. Decka behaved unsatisfactory achieving $41.91 \%$ nematode reduction in the field. Mega NPK recaptured its potency in the field by achieving $50 \%$ reduction in nematode population. Perfect sustained its normal nematicidal action in the field which was around $70 \%$.

\section{b. Foliar sprays treatments}

Data shown in table (4) infer that differences in all abiotic treatments (except Humo plus 40) were significant in reducing root, soil, final population and multiplication rates of $R$. reniformis as compared to check, however some of them showed no significance with each other. IBA was the most efficacious treatment, yet Kenzo was the lowest, Humo plus 40 had no effect at all, in the contrary improved the nematode development and reproduction recording values more than that of the nematode check.

Amino strong elevated its omnipotence to $67.74 \%$ in the field. Cal mix action was inconsistent. It encounted significantly the nematode criteria in the field as compared to check with improper reduction rate.

Feed chem was active achieving $67.98 \%$ in the field, meanwhile UniBor was not, achieving only $18.71 \%$ of reduction.

The plant growth regulator Biogibb beard resemblance to Gibirillic acid treatment in the field obtaining almost the same efficacious rates. Kenzo was not satisfactory effective. Glutamic acid increased its percentages of reductions to more than $50 \%$ in the field. Similar pattern was seen with citric acid treatment which reached its maximum potency in the field. 
Table (3): Reproductivity of Rotylenchulus reniformis as influenced by biotic and abiotic commercial products treated as soil drench under field condition

\begin{tabular}{|c|c|c|c|c|c|c|}
\hline \multirow[b]{2}{*}{ Treatment } & \multirow[b]{2}{*}{ Dose/ plant } & \multicolumn{3}{|c|}{ Nematode counts } & \multirow[b]{2}{*}{$\mathrm{Pf} / \mathrm{Pi}$} & \multirow{2}{*}{$\begin{array}{l}\text { \%change } \\
\text { of Final } \\
\text { population }\end{array}$} \\
\hline & & $\begin{array}{l}\text { Root pop.l } \\
2 \text { gm root }\end{array}$ & $\begin{array}{l}\text { soil pop.l } \\
250 \text { gm soil }\end{array}$ & $\begin{array}{c}\text { Final } \\
\text { population }\end{array}$ & & \\
\hline $\begin{array}{l}\text { Biofertile } \\
\text { (Mixed bacterial solution ) }\end{array}$ & $5 \mathrm{ml} /$ plant & $400.67 \mathrm{~cd}$ & $1656.00 \mathrm{~d}$ & $2056.67 \mathrm{~h}$ & $1.03 \mathrm{~h}$ & -72.69 \\
\hline Pseudomonas fluorescens & $5 \mathrm{ml} /$ plant & $224.67 \mathrm{de}$ & $3646.00 \mathrm{bc}$ & 3870.67 ef & 1.94 ef & -48.59 \\
\hline Serratiamarcescens & $5 \mathrm{ml} /$ plant & $406.00 \mathrm{~cd}$ & $2940.00 \mathrm{c}$ & $3346.00 \mathrm{fg}$ & $1.67 \mathrm{fg}$ & -55.56 \\
\hline $\begin{array}{l}\text { Mycorrhiza } \\
\text { (vesicular arbuscular mycorrhiza) } \\
\text { Algeferet }\end{array}$ & $3 \mathrm{gm} /$ plant & $179.00 \mathrm{de}$ & $4071.00 \mathrm{~b}$ & $4250.00 \mathrm{e}$ & $2.12 \mathrm{e}$ & -43.6 \\
\hline $\begin{array}{l}\text { (Marine algae extract }+ \text { Amino acids }+ \text { plant growth regulator }+ \\
\text { Macro and micro elements) }\end{array}$ & $2 \mathrm{ml} / \mathrm{plant}$ & $625.00 \mathrm{bc}$ & $2041.33 d$ & $2666.33 \mathrm{gh}$ & $1.33 \mathrm{gh}$ & -64.59 \\
\hline $\begin{array}{l}\text { Nemastop } \\
\text { (Plant extracts from Alliums spp. +Titrasulphide Tri- Di- Mono) }\end{array}$ & $2 \mathrm{ml} /$ plant & $117.00 \mathrm{e}$ & $5628.00 \mathrm{a}$ & $5745.00 \mathrm{bc}$ & $2.87 \mathrm{bc}$ & -23.7 \\
\hline $\begin{array}{l}\text { Nile compost } \\
\text { (plant residues ) }\end{array}$ & $5 \mathrm{gm} / \mathrm{plant}$ & $754.00 \mathrm{~b}$ & $5461.67 \mathrm{a}$ & $6215.67 b$ & $3.11 \mathrm{~b}$ & -17.45 \\
\hline $\begin{array}{l}\text { Nema clean } \\
\text { (Amino acids) } \\
\text { Decka }\end{array}$ & $0.5 \mathrm{ml} /$ plant & $837.67 \mathrm{~b}$ & $4259.67 \mathrm{~b}$ & $5097.33 \mathrm{~cd}$ & $2.55 \mathrm{~cd}$ & -32.3 \\
\hline $\begin{array}{l}\text { (Humic acid+ Fulvic acid+ Amino acids+ macro and micro } \\
\text { elements ) }\end{array}$ & $1 \mathrm{ml} /$ plant & $436.67 \mathrm{~cd}$ & $3937.67 \mathrm{~b}$ & 4374.33 de & $2.19 \mathrm{de}$ & -41.91 \\
\hline $\begin{array}{l}\text { Mega N/P/K } \\
(\mathrm{N} 19 / \mathrm{P} 19 / \mathrm{K} 19+\mathrm{Mg} 1 \%)\end{array}$ & $\begin{array}{l}5 \mathrm{gm} / \text { liter } \\
(25 \mathrm{ml} / \text { plant })\end{array}$ & $637.30 \mathrm{bc}$ & $3032.00 \mathrm{c}$ & 3669.30 ef & 1.83 ef & -51.27 \\
\hline $\begin{array}{l}\text { Perfect } \\
\text { (Oxamyl 24\%+ Metalaxyl-M4\%+ Tetramethrin 2\%) }\end{array}$ & $0.4 \mathrm{ml} / \mathrm{plant}$ & $215.30 \mathrm{de}$ & $2094.33 d$ & $2309.63 \mathrm{~h}$ & $1.15 \mathrm{~h}$ & -69.33 \\
\hline Nematode only & & $1281.67 \mathrm{a}$ & $6248.00 \mathrm{a}$ & $7529.67 \mathrm{a}$ & $3.76 \mathrm{a}$ & 0 \\
\hline
\end{tabular}

In each column, values followed by the same letter(s) are not significantly different $(P \leq 0.05)$. 
Table (4): Reproductivity of Rotylenchulus reniformis as influenced by abiotic commercial products treated as foliar sprays under field condition.

\begin{tabular}{|c|c|c|c|c|c|c|}
\hline \multirow[b]{2}{*}{ Treatment } & \multirow[b]{2}{*}{ Dose/ plant } & \multicolumn{3}{|c|}{ Nematode counts } & \multirow[b]{2}{*}{ Pf/Pi } & \multirow{2}{*}{$\begin{array}{c}\text { \%change of } \\
\text { Final } \\
\text { population }\end{array}$} \\
\hline & & $\begin{array}{l}\text { Root pop.l } \\
2 \text { gm root }\end{array}$ & $\begin{array}{c}\text { soil pop.l } \\
250 \mathrm{gm} \text { soil }\end{array}$ & Final pop. & & \\
\hline $\begin{array}{l}\text { Cal mix } \\
\text { (Calcium and Boron carried on organic and amino acids) }\end{array}$ & $0.4 \mathrm{ml} /$ plant & $960.00 \mathrm{bc}$ & $5400.00 \mathrm{bc}$ & $6360.00 \mathrm{~b}$ & $3.18 b$ & -15.53 \\
\hline $\begin{array}{l}\text { Humo plus } 40 \\
\text { (Humic acid }+ \text { macro and micro elements) }\end{array}$ & $0.4 \mathrm{ml} /$ plant & $806.00 \mathrm{cde}$ & $7076.00 \mathrm{a}$ & $7882.00 \mathrm{a}$ & $3.94 \mathrm{a}$ & 4.68 \\
\hline $\begin{array}{l}\text { Amino strong } \\
(\text { Amino and organic acids }+F e+M n+Z n)\end{array}$ & $0.4 \mathrm{ml} /$ plant & 600.00 efg & 1829.00 efg & $2429.00 \mathrm{de}$ & $1.21 \mathrm{de}$ & -67.74 \\
\hline $\begin{array}{l}\text { Feed chem. } \\
\text { (Macro and micro elements) }\end{array}$ & $8 \mathrm{gm} /$ liter & $369.00 \mathrm{hi}$ & 2042.00 efg & 2411.00 de & $1.21 \mathrm{de}$ & -67.98 \\
\hline $\begin{array}{l}\text { UniBor } \\
\text { (Boron carried with vitamins + Gibirilic acid) }\end{array}$ & $0.5 \mathrm{ml} /$ liter & $1123.00 \mathrm{ab}$ & $4998.00 \mathrm{c}$ & $6121.00 \mathrm{~b}$ & $3.06 \mathrm{~b}$ & -18.71 \\
\hline $\begin{array}{l}\text { Biogibb } \\
\text { (Gibirilic acid + Ca+ B+ cytokinin) }\end{array}$ & $0.25 \mathrm{gm} / \mathrm{liter}$ & $555.67 \mathrm{fgh}$ & 2780.00 def & $3335.67 \mathrm{~cd}$ & $1.67 \mathrm{~cd}$ & -55.7 \\
\hline $\begin{array}{l}\text { Kenzo } \\
\text { (cytokinine+ 6-Benzylaminopurine) }\end{array}$ & $0.2 \mathrm{ml} / \mathrm{liter}$ & $832.00 \mathrm{~cd}$ & $5601.67 \mathrm{bc}$ & $6433.67 \mathrm{~b}$ & $3.22 \mathrm{~b}$ & -14.56 \\
\hline Glutamic acid & $1 \mathrm{gm} /$ liter & $253.00 \mathrm{i}$ & $3488.00 \mathrm{~d}$ & $3741.00 \mathrm{c}$ & $1.87 \mathrm{c}$ & -50.32 \\
\hline Citric acid & $2 \mathrm{gm} /$ liter & $565.67 \mathrm{fgh}$ & $1771.00 \mathrm{fg}$ & $2336.67 \mathrm{de}$ & $1.17 \mathrm{de}$ & -68.97 \\
\hline $\begin{array}{l}\text { Nemakill } \\
\text { (Indolylbutric acid } 0.002 \% \text { ) }\end{array}$ & $0.2 \mathrm{ml} / \mathrm{liter}$ & $925.50 \mathrm{bc}$ & $5268.00 \mathrm{bc}$ & $6193.50 \mathrm{~b}$ & $3.10 \mathrm{~b}$ & -17.75 \\
\hline Indole-3 butyric acid & $0.1 \mathrm{gm} / \mathrm{liter}$ & $356.00 \mathrm{hi}$ & $1410.00 \mathrm{~g}$ & $1766.00 \mathrm{e}$ & $0.88 \mathrm{e}$ & -76.55 \\
\hline Indole-acetic acid & $0.2 \mathrm{gm} / \mathrm{liter}$ & 664.00 defg & $3166.67 \mathrm{~d}$ & $3830.67 \mathrm{c}$ & $1.92 \mathrm{c}$ & -49.13 \\
\hline Gibirilic acid & $0.2 \mathrm{gm} / \mathrm{liter}$ & $497.00 \mathrm{gh}$ & $2812.00 \mathrm{de}$ & $3309.00 \mathrm{~cd}$ & $1.65 \mathrm{~cd}$ & -56.05 \\
\hline Salicylic acid & $1 \mathrm{gm} /$ liter & 739.00 cdef & 2514.00 def & $3253.00 \mathrm{~cd}$ & $1.63 \mathrm{~cd}$ & -56.8 \\
\hline Nematode only & & $1281.67 \mathrm{a}$ & $6248.00 \mathrm{ab}$ & 7529.67 a & $3.76 \mathrm{a}$ & 0 \\
\hline
\end{tabular}

In each column, values followed by the same letter(s) are not significantly different $(P \leq 0.05)$. 
Our results reveal that the inducing systemic resistance materials are more constant in reducing the reniform population in the field than any material used, recording the most efficacious rates.

Indole- butyric acid was the highest (76.55\%) reduction and Indole- acetic acid was the lowest. Salicylic acid recorded more than $50 \%$ reduction in the field.

\section{Plant growth response}

Soil drench treatments applied in the experiment (Table 5) indicated to wide variability in plant growth and yield due to commercial agro-products treatment. Generally, significant differences were recorded in yield characters more than growth parameters. Also, differences were significant in some not all criteria within materials in each group and among groups. $R$. reniformis alone inhibited significantly all growth parameters and yield criteria when compared to healthy check.

In case of microbial agents, Serratia marcesens ameliorated significantly shoot fresh weight, number of pods, their fresh and dry weights and number of seeds. But insignificant increase was found in shoot dry weight. No significant enhancement but disorder in some cases (Mycorrhiza and Pseudomonas treatments) when compared with nematode check. In case of Biofertile no significant improvement but disorder in plant fresh and dry weights, but significant increase in yield criteria was found in number of pods, pods dry weight and number of seeds when compared with the nematode check.

Differences between the plant extracts (Nemastop and Nile compost) were significant in improving cowpea yield characters but not shoot parameters, though no significance was found when compared to each other. NPK significantly promoted pods dry weight and number of seeds but not in number of pods and their fresh weight. The nemacide Perfect improved shoot fresh and dry weights but disordered yield characters significantly.

In the amino and organic acids compounds, Nemaclean achieved the highest yield in terms of number of pods, fresh and dry weights of pods and number of seeds and overmatched even healthy check. Decka was the second, which improved significantly shoot parameters, pods dry weights and number of seeds.

Results of foliar sprays of the abiotic treatments in the field (Table 6) indicate to proportional relations between plant growth, yield parameters and the capabilities of commercial materials in reducing nematode population in many cases especially those inducing systemic acquired resistance materials.

No significant improve was observed in Cal mix and Humo plus 40 treatments but disorder in the former treatment. Meanwhile, Amino strong which was highly efficient in hampering $R$. reniformis population, enhanced significantly all parameters, and yield in particular. Feed chem. and UniBor exhibited significant increase in pod dry weight and number of seeds as compared to nematode check with no significance between them. 
Table (5): Cowpea growth and yield as influenced by Rotylenchulus reniformis infection and biotic and abiotic soil drench treatments under field conditions.

\begin{tabular}{|c|c|c|c|c|c|c|c|c|c|c|c|c|c|}
\hline \multirow[b]{2}{*}{ Treatment } & \multirow[b]{2}{*}{ Dose/ plant } & \multicolumn{4}{|c|}{ Plant parameters } & \multicolumn{8}{|c|}{ Yield criteria } \\
\hline & & $\begin{array}{l}\text { plant fresh } \\
\text { weight } \\
\text { (gm) }\end{array}$ & $\begin{array}{c}\% \\
\text { Change }\end{array}$ & $\begin{array}{l}\text { shoot dry } \\
\text { weight } \\
\text { (gm) }\end{array}$ & $\begin{array}{c}\% \\
\text { Change }\end{array}$ & $\begin{array}{l}\text { no. } \\
\text { pods }\end{array}$ & $\begin{array}{c}\% \\
\text { Change }\end{array}$ & $\begin{array}{l}\text { pods fresh } \\
\text { weight } \\
\text { (gm) }\end{array}$ & $\begin{array}{l}\% \\
\text { Change }\end{array}$ & $\begin{array}{l}\text { pods dry } \\
\text { weight } \\
\text { (gm) }\end{array}$ & $\begin{array}{c}\% \\
\text { Change }\end{array}$ & $\begin{array}{l}\text { no. seeds/ } \\
\text { pods }\end{array}$ & I $\quad \%$ \\
\hline $\begin{array}{l}\text { Biofertile } \\
\text { (Mixed bacterial solution ) }\end{array}$ & $5 \mathrm{ml}$ & $135.7 \mathrm{f}$ & -7.8 & $25.7 \mathrm{e}$ & -16.7 & $4.4 \mathrm{de}$ & 88.8 & $6.9 \mathrm{e}$ & 11.4 & $4.9 \mathrm{c}$ & 206.3 & $43.8 \mathrm{bcd}$ & 188.5 \\
\hline Pseudomonas fluorescens & $5 \mathrm{ml}$ & 167.3 ef & 13.7 & 25.9 e & -16.0 & $2.0 \mathrm{fg}$ & -14.2 & $4.2 \mathrm{f}$ & -31.9 & $1.1 \mathrm{~d}$ & -31.3 & $11.0 \mathrm{f}$ & -27.5 \\
\hline Serratiamarcescens & $5 \mathrm{ml}$ & 242.4 bcde & 64.7 & $40.9 \mathrm{bcd}$ & 32.7 & $6.5 \mathrm{ab}$ & 179.0 & $15.4 \mathrm{~b}$ & 149.6 & $8.5 \mathrm{a}$ & 431.3 & $60.5 \mathrm{a}$ & 298.8 \\
\hline $\begin{array}{l}\text { Mycorrhiza } \\
\text { (vesicular arbuscular mycorrhiza) }\end{array}$ & $3 \mathrm{gm}$ & 186.6 def & 26.8 & $40.3 \mathrm{bcd}$ & 30.8 & $1.0 \mathrm{~g}$ & -57.1 & $3.0 \mathrm{f}$ & -51.4 & $1.0 \mathrm{~d}$ & -37.5 & $8.0 \mathrm{f}$ & -47.3 \\
\hline $\begin{array}{l}\text { Algeferet } \\
\text { (Marine algae extract +Amino acids+ plant growth } \\
\text { regulator + Macro and micro elements) }\end{array}$ & $2 \mathrm{ml}$ & 167.7 ef & 13.9 & $29.9 \mathrm{de}$ & -3.0 & $4.0 \mathrm{e}$ & 71.7 & $11.0 \mathrm{c}$ & 78.3 & $4.5 \mathrm{c}$ & 181.3 & $38.5 \mathrm{~d}$ & 153.8 \\
\hline $\begin{array}{l}\text { Nemastop } \\
\text { (Plant extracts from Alliums spp. + Titrasulphide } \\
\text { Tri- Di- Mono) }\end{array}$ & $2 \mathrm{ml}$ & $260.1 \mathrm{bcd}$ & 76.7 & 35.0 cde & 13.6 & $6.0 \mathrm{abc}$ & 157.5 & $9.3 \mathrm{~d}$ & 50.7 & $8.1 \mathrm{ab}$ & 406.3 & $50.0 \mathrm{~b}$ & 229.6 \\
\hline $\begin{array}{l}\text { Nile compost } \\
\text { (plant residues ) }\end{array}$ & $5 \mathrm{gm}$ & $133.2 \mathrm{f}$ & -9.5 & $27.4 \mathrm{de}$ & -11.0 & $5.3 \mathrm{bcd}$ & 128.8 & $9.7 \mathrm{~cd}$ & 57.2 & $7.0 \mathrm{~b}$ & 337.5 & $48.3 \mathrm{bc}$ & 218.6 \\
\hline $\begin{array}{l}\text { Decka } \\
\text { (Humic acid+ Fulvic acid+ Amino acids+ macro } \\
\text { and micro elements ) }\end{array}$ & $1 \mathrm{ml}$ & 361.9 a & 145.9 & $57.7 \mathrm{a}$ & 87.3 & $4.9 \mathrm{cde}$ & 109.0 & $7.4 \mathrm{e}$ & 19.5 & $4.8 \mathrm{c}$ & 200.0 & $41.9 \mathrm{~cd}$ & 176.0 \\
\hline $\begin{array}{l}\text { Nema clean } \\
\text { (Amino acids) }\end{array}$ & $0.5 \mathrm{ml}$ & 216.2 cdef & 46.9 & $36.8 \mathrm{cde}$ & 19.4 & $7.0 \mathrm{a}$ & 200.4 & $21.1 \mathrm{a}$ & 241.5 & $8.4 a b$ & 425.0 & $60.0 \mathrm{a}$ & 295.5 \\
\hline $\begin{array}{l}\text { Mega N/P/K } \\
\text { (N19/P19/K19 + Mg 1\%) }\end{array}$ & $\begin{array}{l}5 \mathrm{gm} / \text { liter } \\
(25 \mathrm{ml} / \text { plant })\end{array}$ & 199.6 def & 35.6 & $56.5 \mathrm{a}$ & 83.3 & $2.3 \mathrm{f}$ & -2.6 & $7.0 \mathrm{e}$ & 13.5 & $4.3 \mathrm{c}$ & 166.9 & $28.9 \mathrm{e}$ & 90.7 \\
\hline $\begin{array}{l}\text { Perfect } \\
\text { (Oxamyl 24\%+ Metalaxyl-M4\%+ Tetramethrin 2\%) }\end{array}$ & $0.4 \mathrm{ml}$ & $300.2 \mathrm{abc}$ & 104.0 & $52.5 \mathrm{ab}$ & 70.5 & $1.0 \mathrm{~g}$ & -57.1 & $4.0 \mathrm{f}$ & -35.1 & $1.0 \mathrm{~d}$ & -35.6 & $9.0 \mathrm{f}$ & -40.7 \\
\hline Nematode only & & $147.2 \mathrm{f}$ & 0.0 & $30.8 \mathrm{de}$ & 0.0 & $2.3 \mathrm{f}$ & 0.0 & $6.2 \mathrm{e}$ & 0.0 & $1.6 \mathrm{~d}$ & 0.0 & $15.2 \mathrm{f}$ & 0.0 \\
\hline Healthy plant & & $315.8 \mathrm{ab}$ & 114.6 & $47.1 \mathrm{abc}$ & 52.9 & $5.5 \mathrm{bcd}$ & 136.1 & $9.7 \mathrm{~cd}$ & 56.7 & $7.3 \mathrm{ab}$ & 356.3 & $40.0 \mathrm{~d}$ & 163.7 \\
\hline
\end{tabular}

In each column, values followed by the same letter(s) are not significantly different $(P \leq 0.05)$. 
Table (6): Cowpea growth and yield as influenced by Rotylenchulus reniformis infection and abiotic foliar spray treatments under field conditions.

\begin{tabular}{|c|c|c|c|c|c|c|c|c|c|c|c|c|c|}
\hline \multirow[b]{2}{*}{ Treatment } & \multirow[b]{2}{*}{ Dose /plant } & \multicolumn{4}{|c|}{ Plant parameters } & \multicolumn{8}{|c|}{ Yield criteria } \\
\hline & & $\begin{array}{l}\text { plant fresh } \\
\text { weight } \\
\text { (gm) }\end{array}$ & $\begin{array}{c}\% \\
\text { Change }\end{array}$ & $\begin{array}{l}\text { shoot dry } \\
\text { weight } \\
\text { (gm) }\end{array}$ & $\begin{array}{c}\% \\
\text { Change }\end{array}$ & $\begin{array}{l}\text { no. } \\
\text { pods }\end{array}$ & $\begin{array}{c}\% \\
\text { Change }\end{array}$ & $\begin{array}{l}\text { pods fresh } \\
\text { weight } \\
\text { (gm) }\end{array}$ & $\begin{array}{l}\mathrm{n} \% \\
\text { Change }\end{array}$ & $\begin{array}{c}\text { pods dry } \\
\text { weight } \\
\text { (gm) }\end{array}$ & $\begin{array}{c}\% \\
\text { Change }\end{array}$ & $\begin{array}{l}\text { no.seeds/ } \\
\text { pods }\end{array}$ & Change \\
\hline $\begin{array}{l}\text { Cal mix } \\
\text { (Calcium and Boron carried on organic and } \\
\text { amino acids) }\end{array}$ & $0.4 \mathrm{ml} /$ plant & $77.2 \mathrm{e}$ & -47.6 & $13.4 \mathrm{~g}$ & -56.5 & $2.0 \mathrm{f}$ & -14.2 & $5.8 \mathrm{f}$ & -6.0 & $2.2 \mathrm{cde}$ & 37.5 & $20.0 \mathrm{fg}$ & 31.8 \\
\hline $\begin{array}{l}\text { Humo plus } 40 \\
\text { (Humic acid + macro and micro elements) }\end{array}$ & $0.4 \mathrm{ml} /$ plant & $170.3 \mathrm{~cd}$ & 15.7 & 28.2 def & -8.4 & $2.3 \mathrm{f}$ & 0.0 & $6.3 \mathrm{f}$ & 2.1 & $1.7 \mathrm{e}$ & 6.3 & $13.0 \mathrm{~g}$ & -14.3 \\
\hline $\begin{array}{l}\text { Amino strong } \\
\text { (Amino and organic acids }+F e+M n+Z n)\end{array}$ & $0.4 \mathrm{ml} /$ plant & t $347.1 \mathrm{ab}$ & 135.9 & $54.6 a b$ & 77.2 & $5.2 \mathrm{cde}$ & 124.5 & $14.6 \mathrm{~cd}$ & 136.6 & $7.2 \mathrm{~b}$ & 350.0 & $46.2 \mathrm{c}$ & 204.8 \\
\hline $\begin{array}{l}\text { Feed chem. } \\
\text { (Macro and micro elements) }\end{array}$ & $8 \mathrm{gm} /$ liter & $145.1 \mathrm{cde}$ & -1.4 & $24.6 \mathrm{efg}$ & -20.2 & $2.8 \mathrm{ef}$ & 18.9 & $5.4 \mathrm{f}$ & -12.5 & $3.1 \mathrm{cde}$ & 93.8 & $19.5 \mathrm{fg}$ & 28.5 \\
\hline $\begin{array}{l}\text { UniBor } \\
\text { (Boron carried with vitamins + Gibirilic acid) }\end{array}$ & $0.5 \mathrm{ml} /$ liter & $91.4 \mathrm{de}$ & -37.9 & $17.1 \mathrm{fg}$ & -44.5 & 3.1 def & 33.1 & 8.9 ef & 43.8 & $4.1 \mathrm{~cd}$ & 154.4 & $35.0 \mathrm{cde}$ & 130.7 \\
\hline $\begin{array}{l}\text { Biogibb } \\
\text { (Gibirilic acid + Ca+ B+ cytokinin) }\end{array}$ & $0.25 \mathrm{gm} / \mathrm{liter}$ & r $342.1 \mathrm{ab}$ & 132.4 & $54.6 \mathrm{ab}$ & 77.2 & $4.5 \mathrm{cdef}$ & 93.1 & $11.9 \mathrm{de}$ & 92.4 & $4.4 \mathrm{c}$ & 176.9 & $19.5 \mathrm{fg}$ & 28.5 \\
\hline $\begin{array}{l}\text { Kenzo } \\
\text { (cytokinine+ 6-Benzylaminopurine) }\end{array}$ & $0.2 \mathrm{ml} /$ liter & $203.6 \mathrm{c}$ & 38.3 & 30.0 def & -2.6 & 3.0 def & 28.8 & $5.8 \mathrm{f}$ & -6.0 & $3.1 \mathrm{cde}$ & 93.8 & $7.0 \mathrm{~g}$ & -53.9 \\
\hline Glutamic acid & $1 \mathrm{gm} /$ liter & 399.9 a & 171.7 & $63.7 \mathrm{a}$ & 106.8 & $6.0 \mathrm{bc}$ & 157.5 & $18.8 \mathrm{~b}$ & 204.7 & $7.8 \mathrm{~b}$ & 387.5 & $61.0 \mathrm{~b}$ & 302.1 \\
\hline Citric acid & $2 \mathrm{gm} /$ liter & $332.8 a b$ & 126.1 & $61.7 \mathrm{a}$ & 100.3 & 3.2 def & 36.1 & 9.7 ef & 56.7 & $4.4 \mathrm{c}$ & 175.0 & 29.2 def & 92.3 \\
\hline Indole-3 butyric acid & $0.1 \mathrm{gm} /$ /iter & $178.5 \mathrm{c}$ & 21.3 & $31.4 \mathrm{de}$ & 1.9 & $9.5 \mathrm{a}$ & 307.7 & $12.6 \mathrm{cde}$ & 104.2 & $11.2 \mathrm{a}$ & 600.0 & $60.0 \mathrm{~b}$ & 295.5 \\
\hline $\begin{array}{l}\text { Nemakill } \\
\text { (Indolylbutric acid } 0.002 \% \text { ) }\end{array}$ & $0.2 \mathrm{ml} /$ liter & $308.3 \mathrm{~b}$ & 109.4 & $48.3 \mathrm{bc}$ & 56.8 & $10.0 \mathrm{a}$ & 329.2 & $28.8 \mathrm{a}$ & 366.8 & $10.6 \mathrm{a}$ & 562.5 & $75.0 \mathrm{a}$ & 394.4 \\
\hline Indole-acetic acid & $0.2 \mathrm{gm} /$ /iter & $166.1 \mathrm{~cd}$ & 12.8 & $30.7 \mathrm{de}$ & -0.3 & 3.0 def & 28.8 & 9.5 ef & 53.5 & $2.1 \mathrm{de}$ & 29.4 & $21.8 \mathrm{efg}$ & 43.5 \\
\hline Gibirilic acid & $0.2 \mathrm{gm} /$ /iter & $223.1 \mathrm{c}$ & 51.6 & $38.8 \mathrm{~cd}$ & 25.9 & $2.5 \mathrm{f}$ & 7.3 & $6.8 \mathrm{f}$ & 9.7 & $2.1 \mathrm{cde}$ & 33.1 & $15.5 \mathrm{fg}$ & 2.2 \\
\hline Salicylic acid & $1 \mathrm{gm} /$ liter & $169.6 \mathrm{~cd}$ & 15.2 & 30.4 def & -1.4 & $8.0 a b$ & 243.4 & $16.1 \mathrm{bc}$ & 160.9 & $10.8 \mathrm{a}$ & 575.0 & $77.5 \mathrm{a}$ & 410.9 \\
\hline Nematode only & & 147.2 cde & 0.0 & $30.8 \mathrm{de}$ & 0.0 & $2.3 \mathrm{f}$ & 0.0 & $6.2 \mathrm{f}$ & 0.0 & $1.6 \mathrm{e}$ & 0.0 & $15.2 \mathrm{fg}$ & 0.0 \\
\hline Healthy plant & & $315.8 \mathrm{~b}$ & 114.6 & $47.1 \mathrm{bc}$ & 52.9 & $5.5 \mathrm{~cd}$ & 136.1 & $9.7 \mathrm{ef}$ & 56.7 & $7.3 \mathrm{~b}$ & 356.3 & $40.0 \mathrm{~cd}$ & 163.7 \\
\hline
\end{tabular}


Kenzo, Biogibb and Gibirillic acid improvements were not significantly different with the nematode check in all growth and yield criteria, however Biogibb improved fresh and dry weights of either shoot and pods. The SAR treatments differed in their significance in shoot parameters as compared with the check, improve was significant in yield criteria especially pods numbers, pods dry weights and number of seeds. Glutamic, Nemakill and salicylic acid were the most conspicuous.

\section{Discussion}

In field experiment, the microbial agents, however they are rhizoorganisms varied in their effectiveness against $R$. reniformis. (Biofertile \& Algeferet) were highly effective, others achieved less than 50\% efficacy (Pseudomonas fluorescence and Mycorrhiza). Biofertile was rated as the best in the field as it accomplished the highest suppressive effects. The fluctuating malignant actions of microbial products in field impose the importance of different environmental factors that affect their activities in plant rhizosphere (Stirling,1991 and Zuckerman et al., 1993). Biofertile and Serratia marcescens could be recommended as candidates for R.reniformis integrated management programs.

There were no direct proportional effects between capability of microbes to reduce nematode reproduction and improvement of growth criteria.

Commercial compounds composed of microbes are now available in the Egyptian market and acceptable as an alternative for nematicides but they can not stand alone as a control procedure, but it should be involved as an element of complementary program for nematode management.

The addition of products containing amino and organic acids as soil drench are dependent on the compound nature, Decka efficacy ended with $41.91 \%$ in the field, meanwhile, Nemaclean was lower. Amino strong was more efficient in the field than any other mineral compound. Humo plus 40 was not effective at all.

Generally, the foliar spray of abiotic compound was more effective in reducing nematode population than that used as soil drench.

Nemastop followed by Nile compost achieved unsatisfactory reductions. While up word in Algeferet efficacy was recorded to reach highest reduction among all botanicals. Several factors such as nematode host status of the candidate plants, timing of incorporation into the soil, phytotoxicity to crop, stability of nematicidal compounds in the soil and soil type affect plant extracts efficacy. Nematode control by plant preparations containing nematicidal compounds seems to be more effective in light soils such as sandy than heavy soils, because in the later, active compounds may be absorbed by the clay and inactivated (Oka, 2010). 
In some experiments composts showed activity against root knot and root lesion nematodes (LaMondia et al.,1999 \& Everts et al., 2006) but in others, composts affected nematodes only slightly, if at all (McSorley and Gallaher 1995, 1996). In a long term experiment, application of compost increased total yield of potato and grain yields. Certain microorganisms in composts are thought to play a suppressive role to nematodes (Sharon et al., 2001; Kokalis- Burelle et al., 2003; Mekete et al., 2009). Other studies indicated that nitrogenous compounds rather than microorganisms contribute to nematode suppression (Oka and Yermiyahu, 2002 \& Raviv et al., 2005).

Ammonia released from organic materials during microbial decomposition plays an important role in nematode control. Anhydrous and aqueous ammonia, urea and other ammonium compounds have been used for nematode control (Eno et al.,1955 \& Walker, 1971). The nematicidal activity of ammonia greatly depends on soil $\mathrm{pH}$, humidity and temperature.

McSorley (2011) summarized reasons for variability in organic amendments performance and boasting tolerance of plants to infection in usage of many different materials, results may differ vary with nematodes and environmental factors, most organic amendments are fertilizers, greenhouse results may differ from field conditions, amendments effects not limited to plant parasitic nematodes. Several authors (Csizinszky,1999 and Noling,1997) observed that composts on mulches did not suppress root-knot nematodes. However despite lack of nematode control, tomato yield in compost- amended plots was $196 \%$ of the yield in control plots.

The systemic acquired resistance commercial materials applied on foliage activated nematode reductions. The exogenous application of IAA or IBA as well as amino acid containing formulations may restrict nematode biology which ultimately acquire some resistance to treated cowpea against $R$. reniformis. Our results are supported with those of $\mathrm{Yu}$ and Zhena (2007). Khare and Arora (2010) attributed the biocontrol activity of the fluorescent Pseudomonas to their ability to produce IAA.

IAA and IBA are considered pesticide derivatives (Omar and Muneer, 2005). They reported that IBA has been classified as a biocontrol pesticide because it is similar in structure and function to the naturally occurring plant growth Indol-3-acetic acid. Also, B amino-butyric acid is known as chemical plant defense activator and has been used successfully to induce resistance against a wide spectrum of pathogens (Sahebani et al., 2011).

Concerning the commercial inorganic fertilizers (Mega NPK, Feed chem, UniBor) were highly fluctuating in their action in the field. However, they were significantly effective in reducing the nematode counts. UniBor was the lowest in the field. Feed chem controverted Uni Bor. Efficacy of NPK achieved more than $50 \%$ efficacy in the field. Despite that they all succeeded significantly in minimizing the 
nematode population. Concerning NPK, reports of Waceke and Waudo (1993), Bamelet al. (2003), Coyne et al. (2004), Kheir et al. (2009) and Farahat et al. (2010) are congenial with our findings.

It is interesting to notice that Cal mix disorded plants in all experiment and Mega NPK recorded best improvement in plant fresh, dry weights and yield criteria, however reduction in nematode population was the lowest. Sharma and Khan (1995) reported that tomato given potassium alone were heavily infested with $M$. incognita also high levels of $\mathrm{P}$ could not overcome the deleterious effects of $R$. reniformis on cotton yield and there was no interaction between levels of potassium and $R$. reniformis on cotton growth. In contrast, in cowpea field study, NPK decreased the population of $M$. incognita root galling and in soil (Egunjobi and Olaitan 1986). Similar results were obtained by Ahmed et al. (1991) and Olowe (2012). The latter reported that $M$. incognita galling in the field was reduced by $53 \%$ by NPK and the fertilizer has a nematicidal effect. NPK has been observed to boost tolerance of plants to diseases, cause nematode mortality (Melaka Barhan et al., 1997).

Our results concerning cowpea growth could be summarized as follows, the applications of biotic and abiotic commercial agro-products resulted in differences in their performance. Foliar application treatments achieved stimulatory effects on plant growth more than soil application and growth parameters were ameliorated.

There were direct proportion effects between potency of different sprayed materials in reducing nematode population and growth response in many cases, but was to some extent in soil. The compounds used to induce systemic acquired resistance exhibited more enhancements in plant growth criteria and were more consistent in reducing the nematode population. Biofertile and Algeferet were the eminent soil treatments applied in the field, simultaneously IBA and Amino strong as foliar.

The relation between the material efficacy and its cost-effectiveness should be considered in any nematode management program whereby applying more than one material together may express their potentiality to surpass any chemical nematicide, achieve ultimate performance of plants and transcend environmental hazards.

This research recommend that under comprehensive nematode management programs, the best available alternative lies in the complimentary use of biofertilizers and organic /inorganic materials in suitable combinations with SAR material which together surpass the nemacide effects on nematode reduction. This ensures high productivity and at the same time yields good soil health. 


\section{References}

Abid, M.; Zaki, M. J.; Khan, Q. and Abdul Sattar (2005). Use of marine algae for the management of root-knot nematode (Meloidogyne javanica) in okra and tomato plants. int. j. phycol. phycorhem, 1(2), 187-192.

Ahmed, S. S.; Kandil, M. M. and Al-Ansi, N. A. (1991). Effect of some fertilizers on development of Meloidogyne incognita and growth of cowpea. Annals of Agric. Sci. Moshtohor, 29 (3):1215-1220.

Akhtar, M., and Malik, A. (2000). Roles of organic soil amendments and soil organisms in the biological control of plant-parasitic nematodes: a review. Bioresource Technology, 74(1), 35-47.

Bamel, V.; Verma, K.K.; Gupta, D.C.; Singh, R.V.; Pankajdhawan, S.C. and Gaur, H.S. (2003). Effect of different fertilizer forms on Meloidogyne incognita infecting mungbean (Vigna radiate). Proceedings, of national symposium on Biodiversity and Management of nematodes in Cropping systems for sustainable Agric., Jaipur, India., 240 - 244.

Bationo, A.; Hartemink, A. E.; Lungo, O.; Naimi, M.; Okoth, P.; Smaling, E. M. A. and Thiombiano, L. (2006). African soils: their productivity and profitability of fertilizer use: background paper for the African Fertilizer Summit 9-13th June. Abuja, Nigeria. IFDC.

Coyne, D. L.; Sahrawa, K. L. and Plowright, R. A. (2004). The influence of mineral fertilizer application and plant nutrition on plant parasitic nematodes in upland and low land rice in Cote Divoire and its implications in long-term Agricultural Research trials. Experimental Agriculture 40, 245-256.

Csizinszky, A. A. (1999). Yield and nutrient uptake of 'Capistrano'bell peppers in compost-amended sandy soil. In Proceedings of the Florida State Horticultural Society, 112: 333-337.

Dar, M. H. and Reshi, Z. A. (2017). Vesicular Arbuscular Mycorrhizal (VAM) Fungias a Major Biocontrol Agent in Modern Sustainable Agriculture System. Russian Agricultural Sciences. 43 (2): 138-143.

Egunjobi, O. A. and Olaitan, J. O. (1986). Response of Meloidogyne incognita infected cowpea to some agro- waste soil amendments. Nematropica 16, 33-43.

El-Nagdi, W. M. A. E., and Youssef, M. M. A. (2013). Comparative efficacy of garlic clove and castor seed aqueous extracts against the root-knot nematode, Meloidogyne incognita infecting tomato plants. Journal of Plant Protection Research, 53(3): 285-288.

Eno, C. F.; Blue, W. G. and Good, J. M. (1955). The Effect of Anhydrous Ammonia on Nematodes, Fungi, Bacteria, and Nitrification in Some Florida Soils 1. Soil Science Society of America Journal, 19(1): 55-58. 
Everts, K. L.; Sardanelli, S.; Kratochvil, R. J.; Armentrout, D. K. and Gallagher, L. E. (2006). Root-knot and root-lesion nematode suppression by cover crops, poultry litter, and poultry litter compost. Plant disease, 90(4): 487492.

Farahat, A.A.; Al-Sayed, A.A. and Mahfoud, N.A. (2010). Compost and other organic fertilizers in the scope of root-knot nematode reproduction and control. Egyptian Journal of Agronematology, 9(1): 18-19.

Jones, J.T.; Haegeman, A.; Danchin, E.G.; Gaur, H.S.; Helder, J.; Jones, M.G.; Kikuchi, T.; Manzanilla-López, R.; Palomares-Rius, J.E.; Wesemael, W.M. and Perry, R.N. (2013). Top 10 plant-parasitic nematodes in molecular plant pathology. Mol Plant Pathol. 14(9):946-61.

Khare, E., and Arora, N. K. (2010). Effect of indole-3-acetic acid (IAA) produced by Pseudomonas aeruginosa in suppression of charcoal rot disease of chickpea. Current microbiology, 61(1), 64-68.

Kheir, A. M.; Al-Sayed, A. A. and Saeed, M. R. (2009). Suppressive effects of inorganic fertilizers on $M$. incognita infecting soybean. Egyptian Journal of Agronematology,7(1): 9-19.

Kokalis-Burelle, N.; Vavrina, C. S.; Reddy, M. S. andKloepper, J. W. (2003). Amendment of muskmelon and watermelon transplant media with plant growth-promoting rhizobacteria: Effects on seedling quality, disease, and nematode resistance. HortTechnology, 13(3): 476-482.

LaMondia, J. A.; Gent, M. P. N.; Ferrandino, F. J.; Elmer, W. H. \& Stoner, K. A. (1999). Effect of compost amendment or straw mulch on potato early dying disease. Plant Disease, 83(4), 361-366.

Luangkhot, J. (2016). Integrated Pest Management Systems can be used for Promoting Plant Development in Upland Cotton to Combat Reniform Nematode Losses.Master's thesis. Auburn, Alabama pp.54.

Mahfoud, N. A. (2015). Comparing the biochemical changes and defense mechanisms in plants infected with the root-knot and other nematode species. Ph.D. Thesis Fac. Agric., Cairo Univ., pp.218.

McSorley, R. (2011). Overview of organic amendments for management of plantparasitic nematodes, with case studies from Florida. Journal of Nematology, 43(2), 69-81.

McSorley, R. and Gallaher, R. N. (1995). Effect of yard waste compost on plantparasitic nematode densities in vegetable crops. Journal of Nematology, 27(4S), 545- 549.

McSorley, R. and Gallaher, R. N. (1996). Effect of yard waste compost on nematode densities and maize yield. Journal of Nematology, 28(4S):655660. 
Mekete, T.; Hallmann, J.; Kiewnick, S. and Sikora, R. (2009). Endophytic bacteria from Ethiopian coffee plants and their potential to antagonize Meloidogyne incognita. Nematology, 1(1):117-127.

Melaka Barhan, H.; Bird, G.W. and Gore, R. (1997). Inpact of plant nutrition of Pratylenchus penetrans infection of Prunusavium root stocks Journal Nematology 29, 381-388.

Montasser, S. A.; El-Wahab, A. E. A.; Abd-Elgawad, M. M. M.; Abd-El-Khair, H.; Koura, F. F. H. and Hammam, M. M. A. (2012). Effects of some fungi and bacteria as bio-control agents against citrus nematode Tylenchulus semipenetrans Cobb. Journal of Applied Sciences Research.5436-5444.

Noling, J. W. (1997). Plant resistance and soil amendments in Florida tomato and pepper. Annual International Conference on Methyl Bromide Alternatives, USA.

Oka, Y. (2010). Mechanisms of nematode suppression by organic soil amendments-a review. Applied Soil Ecology, 44(2), 101-115.

Oka, Y. and Yermiyahu, U. (2002). Suppressive effects of composts against the root-knot nematode Meloidogyne javanica on tomato. Nematology, 4(8), 891-898.

Olowe, T. O. (2012). The effect of fertilizer (NPK) on infestation of Meloidogyne incognita on cowpea Vigna unguiculata. British journal of science. 6(2):2542.

Omar, M. and Muneer, M. (2005). Comparative photocatalytic study of two selected pesticide derivatives, Inole-3-acetic acid and Indole-3-buteric acid in aqeous suspensions of titanium dioxide. Journal of Hazard Mater, 11(13): 219-227.

Patil, J. and Sharma, M. K. (2016). Management of reniform nematode, Rotylenchulus reniformis by soil application with bioagents on cowpea. Life Sciences International Research Journal. 3(1); 9-13.

Raviv, M.; Oka, Y.; Katan, J.; Hadar, Y.; Yogev, A.; Medina, S. andZiadna, H. (2005). High-nitrogen compost as a medium for organic container-grown crops. Bioresource technology, 96(4): 419-427.

Robinson, A. F.; Inserra, R. N.; Caswell-Chen, E. P.; Vovlas, N. and Troccoli, A. (1997). Rotylenchulus species: identification, distribution, host ranges, and crop plant resistance..Nematropica 27: 127-180.

Robinson, A.F.; Akridge, R.; Bradford, J.M.; Cook, C.G.; W. S.; Kirkpatrick, T. L.; Lawrence, G. W.; Lee, G.; McGawley, E. C.; Overstreet, C.; Padgett, B.; Rodríguez-Kábana, R.; Westphal, A. and Young, L. D. (2005). Vertical Distribution of Rotylenchulus reniformis in Cotton Fields. Journal of Nematology 37(3):265-271. 
Sahebani, N.; Hadavi, N. S. and Zade, F. O. (2011). The effects of $\beta$-amino-butyric acid on resistance of cucumber against root-knot nematode, Meloidogyne javanica. Actaphysiologiaeplantarum, 33(2): 443-450.

Sharma, G.C. and Khan, M.L. (1995). Effect of N.P.K. fertilizers on Meloidogyne incognita infestation and plant growth in tomato. Indian Journal of Nematology, 25 (2): 147-149.

Sharon, E.; Bar-Eyal, M.; Chet, I.; Herrera-Estrella, A.; Kleifeld, O. and Spiegel, Y. (2001). Biological control of the root-knot nematode Meloidogyne javanica by Trichoderma harzianum. Phytopathology, 91(7), 687-693.

Stirling, G. R. (1991). Biological control of plant-parasitic nematodes. Wallingford, UK: CAB International.

Waceke, J.W. and Waudo, S.W. (1993). Effect of soil amendments on pathogenicity of Meloidogyne incognita on okra. International Journal of Pest Management, 39 (4): 385-389.

Walker, J.T. (1971). Populations of Pratylenchus penetrans relative to decomposing nitrogenous soil amendments. Journal of Nematology, 3(1): 43-49.

Youssef, M. M. A. and Lashein, A. M. S. (2014). Induction of systemic acquired resistance in date palm plants against certain plant parasitic nematodes by some chemicals. Pakistan Journal of Nematology, 32(2):131-136.

Youssef, M.M.A and Eissa, M.F.M. (2014). Biofertilizers and their role in management of plant parasitic nematodes. A review. E3 Journal of Biotechnology and Pharmaceutical Research Vol. 5(1): 001-006.

Yu, T. and Zhena, X. D. (2007). Indole-3-acetic acid enhances the biocontrol of Penicillium expansum and Botrytis cinerea on pear fruit by Cryptococcus laurenti. FEMS Yeast Research 7(3): 459-464.

Zeeshan, M. A.; Khan, I.; Shah, B.; Naeem, A.; Khan, N.; Ullah, W. and Iqbal, M. (2016). Study on the management of Ralstonia solanacearum (Smith) with spent mushroom compost.Journal of Entomology and Zoology Studies, 4(3): 114-121.

Zuckerman, B.M.; Dicklow, M. B. and Acosta, N. (1993). A strain of Bacillus thuringiensis for the control of plant parasitic nematodes. Biocontrol Science and Technology, 3: 41-46. 
مقارنة كفاءة المنتجات التجارية الزراعية الحيوية وغير الحيوية ضد النيماتودا الكلوية تحت ظروف الحقل

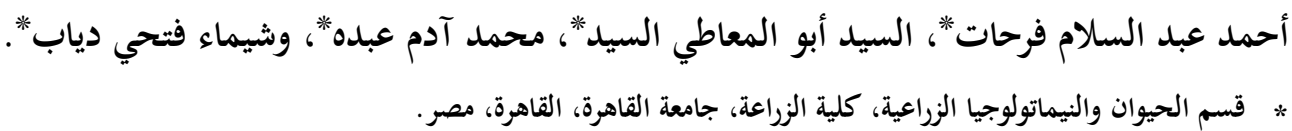

في تجربة حقلية تم اختبار اثنى عشر منتجًا تجاريًّ زراعيًّا عوملت في التربة، وأربعة

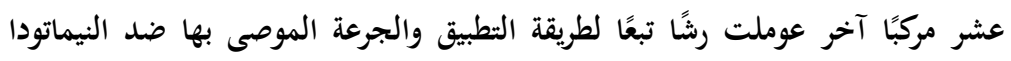

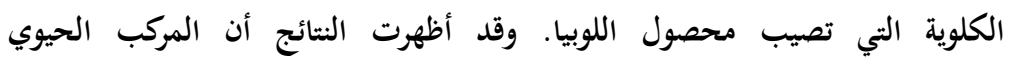

Biofertile

مركبي Algeferet Perfect عندما استخدما أيضا "ريًّا" في التربة وذلك بالمقارنة

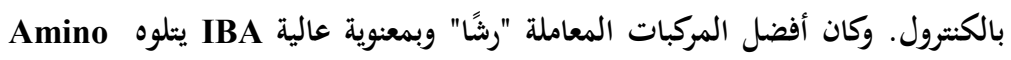

Feed Chem و دون فروق معنوية بينهم. وأوضحت التجربة أن المواد التجارية

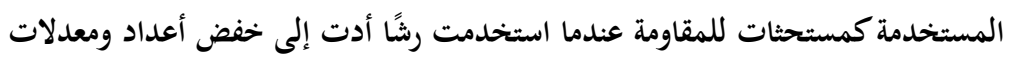

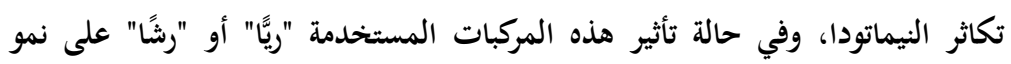

نباتات اللوبيا ومحصولها قد سجل مركب Mega NPK أفضل النتائج، وبصفة عامة فِإِان

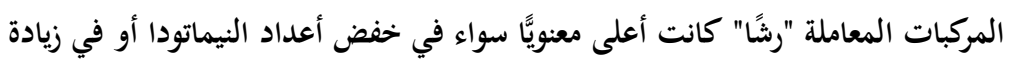
نمو النبات وإنتاجيته إذا ما قورنت بالكنترول وخاصة تلك المركبات المستخدمة كمستحثات للمقاومة الجهازية للنبات عن المركبات المستخدمة في التربة. 